\title{
OC01 - The effect of an Interactive Therapeutic Play Training Program on the anxiety levels of children having heart surgery and their mothers
}

\author{
Ayşegül Elmas Coşantürk (Turkey); Duygu Gözen (Turkey)²
}

\begin{abstract}
${ }^{1}$ Kartal Koşuyolu High Specialized Education and Research Hospital; ${ }^{2}$ Istanbul University Florence Nightingale Faculty of Nursing
\end{abstract}

Theme: Child protection and managing risk.

Keywords: Anxiety, Beck Anxiety Inventory, congenital heart disease, therapeutic play.

Research was conducted using randomized controlled and experimental methods in order to determine the impact of Interactive Therapeutic Play Training Program applied for preparation of children's heart disease operation on child's and mother's post-operative anxiety levels. The sample consisted of 23 experimental and 20 control child-mother pairs who were hospitalized between April-September 2015 with Congenital Heart Disease in the six to 12 years age group. The day before the operation, children in the experimental group with their mother were given 'Interactive Therapeutic Play Education Program'. Data was obtained with an Information form, Anxiety Inventory for Children and Beck Anxiety Scale.

Results: $55 \%$ of children were girls, while $100 \%$ of them were primary school students. As a result, anxiety levels of children and mothers in the preoperative period were similar, whereas in the postoperative period State Anxiety Inventory for Children and Beck Anxiety Inventory scores for the experimental group were found to be lower than control group $(\mathrm{p}<\cdot 0,05)$.

\section{OC02 - Promoting the university research of paediatric nurses}

\author{
Madeleine Collombier (France) ${ }^{1}$; Emilie Courtois (France) ${ }^{1}$; \\ Karine Renaudie (France) ${ }^{1}$; Catherine Thomyre (France ${ }^{1}$
}

\begin{abstract}
${ }^{1} A N P D E$
Theme: Nursing education, management and leadership.

Keywords: Paediatric nursing education, research, scientific publication.

While training to obtain a university degree in addition to the state paediatric nursing diploma, French paediatric nurses conduct research studies, which are not identified nor highlighted.
\end{abstract}

We aim to create a network of paediatric nurses with a university degree, in order to highlight their research.

We conducted a national survey, with questionnaires from June to December 2013, to numerate paediatric nurses with university degrees. Sixty-six paediatric nurses with at least one university degree responded. The more frequent diploma was a one-year university degree in the area of nursing; three studies out of 72 have been published. 